\title{
Robotic surgery: Evolution, current status \& future perspectives
}

\author{
Murtaza Akhtar ${ }^{1, *}$, Divish Saxena ${ }^{2}$ \\ ${ }^{\mathbf{1}}$ Professor and Head, ${ }^{2}$ Assistant Professor, Dept. of Surgery, NKP Salve Institute of Medical Sciences and Research Center, \\ Digdoh Hills, Hingna Road, Nagpur, India \\ *Corresponding Author: \\ Email: murtazaakhtar27@gmail.com
}

\section{Introduction}

"Primum non nocre" is a Latin phrase that means "first, to do no harm" dates back to Hippocratic Oath, is one of the principle percepts of bioethics that all healthcare students and professionals are taught in medical school throughout the world. The same principle is followed by surgeons worldwide to perform surgeries doing minimum tissue damage and post operative pain to their patients. Evolution is a continuous process and the surgical techniques are constantly being modified. The advent of Laparoscopy or Minimal Access Surgery was a boon to many a patients giving them early recovery and recumbence. Robotically assisted surgery was developed to overcome the pre existing limitations of Minimal Access Surgery i.e. 2D vision, dexterity, tremors, freedom and rotation of movements, surgical précising and accuracy and to enhance the capabilities of surgeons performing open surgery. ${ }^{1}$

In robotic surgery, the surgeon uses a direct telemanipulator which is a remote manipulator that allows the surgeon to perform the normal movements associated with the surgery whilst the robotic arms carry out those movements using end-effectors and manipulators to perform the actual surgery on the patient. $^{2}$ One of the advantage of using the computerised method is that the surgeon does not have to be present by the side of the patient, but can be anywhere in the world, leading to the possibility for remotely carrying out surgery. Due to robotic use, the surgery is done with precision, miniaturization, smaller incisions; decreased blood loss, less pain, and quicker healing time. Articulation beyond normal manipulation and three-dimensional magnification helps resulting in improved ergonomics. Due to these techniques there is a reduced duration of hospital stays, blood loss, transfusions, and use of pain medication. Another advantage is that the naturally occurring hand tremors are filtered out by the robot's computer software. The existing open surgery technique has many flaws like limited access to surgical area, long recovery time, long hours of operation, blood loss, surgical scars and marks.

The development of robotic systems was carried out by SRI International and Intuitive Surgical with the introduction of the da Vinci Surgical System and Computer Motion with the AESOP and the ZEUS robotic surgical system. ${ }^{3}$ The first robotic surgery took place at The Ohio State University Medical Centre in
Columbus, Ohio under the direction of Robert E. Michler. Examples of using ZEUS include a fallopian tube reconnection in July 1998, a beating heart coronary artery bypass graft in October 1999, and the Lindbergh Operation, which was a cholecystectomy performed remotely in September 2001. In 2000, the da Vinci Surgical system was approved by FDA for general surgery procedures.

The da Vinci Surgical system is based on Master- Slave concept and has three components; ${ }^{4}$

a. Surgeon's console is the area where the surgeon sits, views and operates using tele-manipulators.

b. Robotic (Patient's) cart has 4 arms mounted on movable wheel base. One arm is for camera port whereas each of the other three arms is provided with "endowrist" that gives 7 degree of freedom movement to the surgeon.

c. Vision Cart provides true 3-D image and binocular vision.

The surgery is started by 'docking' the robotic cart over patient's body area where the surgery has to be performed. When there is a need of conversion to open surgery or after completion of procedure the arm are disengaged and is termed as 'undocking'. Robotic surgery systems are now being used by almost all surgical disciplines ${ }^{5}$ with a spectrum of surgeries ranging from cardiovascular procedures ${ }^{6}$ like coronary bypass, septal, valvular deformities correction, neurosurgical procedures, gynaecology ${ }^{7}$ and paediatric surgery procedures. Many of the urological procedures ${ }^{8}$ like radical prostatectomy, retro peritoneal lymph node dissection and nephrectomy are being carried out by more surgical precision adhering to basic oncological principles. The role of robotics in general and gastrointestinal surgery includes colonic and rectal oncological procedures, cholecystectomy, gastric surgeries, achlasia cardia and Bariatric surgery procedures. Now, even transplant surgeries and organ retrieval surgeries are carried out by robotics.

The biggest short coming of Robotic surgery systems today is the cost involved. Robotic surgery involves significantly higher costs in terms of acquisition, maintenance and recurring instrument costs in comparison to conventional laparoscopic or open procedures. The initial cost of robot today in India ${ }^{9}$ ranges from Rs. 7 to 9 crores. Each instrument costs 
about Rs. 135,000 and is programmed to last for maximum ten uses. Moreover, it has also been seen that learning curve is steep and fewer centers are available for training in India. The other major disadvantage is absence of haptic or tactile feedback, which is an area of concern.

The research is going on to incorporate all patient data like ultrasound, CT, MRI, etc. into robotic system to create a "virtual patient" and rehearse the surgery on virtual patient before actual surgery. Development of real time high speed data transfer technology will allow for Telerobotic Surgery ${ }^{10}$ where a surgeon sitting on console at one continent can operate a patient thousands of miles away on some other remote continent. Development of newer instruments and mass production of instruments may lower the cost of robotic procedures and will make it more affordable for a common man.

The numerous technological innovations are at the horizon that will render the concept of Robotic surgery to stay forever and will become an integral part of every operation theatre sooner or later.

\section{References}

1. Gerhardus D. Robot-assisted surgery: the future is here. Journal of Healthcare Management July-August 2003;48(4):242-51.

2. O'Toole, Bouazza-Marouf K, Kerr D, Gooroochurn M, Vloeberghs M. A methodology for design and appraisal of surgical robotic systems. Robotica 2009;28(2):297310.

3. Ballantyne GH. Robotic surgery, telerobotic surgery, telepresence, and telementoring. Review of early clinical results. Surg Endosc 2002 Oct;16(10):1389-402.

4. Hazey JW, Melvin WS. Robot-assisted general surgery. Semin Laparosc Surg 2004Jun;11(2):107-12.

5. Talamini MA, Chapman S, Horgan S, Melvin WS. A prospective analysis of 211 robotic-assisted surgical procedures. Surgical Endoscopy October 2003;17(10):1521-24.

6. McConnell PI, Schneeberger EW, Michler RE. History and development of robotic cardiac surgery. Problems in General Surgery 2003;20(2):20-30.

7. Liu H, Lawrie TA, Lu D, Song H, Wang L, Shi G. Robotassisted surgery in gynaecology. Cochrane Database Syst Rev 2014;12:CD011422.

8. Finkelstein J, Eckersberger E, Sadri H, Taneja SS, Lepor H, Djavan B. Open versus laparoscopic versus RobotAssisted laparoscopic prostatectomy: The European and US Experience. Reviews in Urology 2010;12(1):35-43.

9. Kumar Arvind, Asaf Belal Bin, Balasubramanium V, Jindal Tarun. Robotic Surgery: Current Status. Recent advances in Surgery; Roshan Lal Gupta, Vol 13; Chapter $1 ; 1-23$.

10. Sandor Jozsef, Haidegger Tamas, Benyo Zoltan. Surgery in space: The future of Robotic Telesurgery. Surgical Endoscopy 2012;26(1):681-90. 